\title{
Éditorial
}

\section{HISTORIQUE \\ DE L'ALIMENTATION EN EAU POTABLE DE LA VILLE DE PARIS}

De Babylone au Pays Dogon, les Hommes ont toujours chargé l'eau de symboles: sources, mère, déluge, chemin du sacré, fontaine des philosophes. L'eau révèle des besoins et des désirs conscients ou inconscients.

L'eau, à l'époque de l'Homme primitif, était à portée de main et assurait facilement, entre autres, sa survie. Il en est tout autrement à l'heure actuelle où l'eau d'alimentation n'est plus si abondante et doit souvent être acheminée jusqu'à l'Homme par d'importants ouvrages.

Paris, ou plus exactement La Lutèce Gauloise puisait directement son eau dans le fleuve pour alimenter ses habitants des deux îles, actuellement de la Cité et de Saint-Louis.

Ce n'est guère que vers le $\mathbf{1}^{\text {er }}$ siècle après $\mathrm{J}$.-C. que le tout premier aqueduc fut construit par les Romains pour alimenter en eau les thermes, situés dans le bas de la rue Saint-Jacques, à la place de l'actuel Collège de France. Il s'agissait d'une simple rigole à ciel ouvert avec une pente régulière, de 45 mètres par $\mathrm{km}$ qui prenait l'eau aux environs de Rungis et l'amenait par le flanc sud de la vạllée de Rungis, puis le flanc droit de la vallée de la Bièvre, par là rue Saint Jacques et aboutissait enfin aux bains publics dans le bas de cette rue. Le débit de l'eau était de $2500 \mathrm{~m}^{3}$ par jour 
Il faut ensuite attendre le $\mathrm{XI}^{\mathrm{c}}$ siècle pour voir la pousuite les travaux d'aménagement de la source Savies au fond de la vallée de Ménilmontant, au sein de l'Abbaye de Saint-Martin-des-Champs. Les eaux de cette source sont amenées jusqu'à Paris par un aqueduc dit, de Belleville, maçonné et recouvert d'une voûte en forme de berceau. Les moines décidèrent de laisser couler l'excès de l'eau par une fontaine située contre le mur extérieur de leur abbaye. C'était la première fontaine publique appelée plus tard, Fontaine de Vertbois.

Au XII ${ }^{e}$ siècle, l'aqueduc du Pré-Saint-Gervais fut l'œuvre des moines de l'abbaye de Saint-Lazare située au pied de Montmartre. Les moines firent des travaux, de captage de la source à l'aide de drains enterrés et de creusement d'un bassin de recueillement des eaux. Là encore, les moines créèrent une fontaine à l'extérieur du prieuré destinée à pourvoir aux besoins en eau potable de la population. C'était la première fontaine intra-muros du futur Paris.

C'est en 1182 que le roi Philippe Auguste rachète la foire "saint Laurent" appartenant au Prieuré de Saint-Lazare et devient par cette occasion propriétaire d'une partie de ces eaux. Il fit alors, réaliser une autre fontaine au centre de la place des Halles pour distribuer ces eaux.

C'est de cette époque et par la volonté de Philippe Auguste que les Prévôts des Marchands et des Échevins vont devoir s'occuper de l'alimentation en eau potable des habitants de Paris et créer plusieurs fontaines à travers la capitale. Ces nouveaux responsables, dès la fin du XIII" siècle et jusqu'à la prise de la Bastille, étaient considérés comme des officiers municipaux. Ils dirigeaient les affaires de la ville et notamment celle des eaux. Ils étaient élus pour deux années à la simple pluralité des voix.

En deux siècles, Belleville était devenue le château d'eau de la capitale et allait le rester durant cinq siècles.

Le XVI" siècle est un siècle noir pour les eaux de Paris: guerres multiples provoquant la détérioration des réseaux, grande sécheresse de 1538 à 1559, concessions particulières, usurpations des sources. En 1553 Paris compte 26000 habitants et chaque habitant peut disposer de 1 litre d'eau par 24 heures.

Henri IV eut une grande influence sur le devenir des projets d'alimentation en eau potable, sans en voir d'ailleurs toutes les réalisations de son vivant. Il redonne de grands pouvoirs aux prévôts des marchands pour reconstituer les fontaines dans leur splendeur ancienne, réprimer le détournement d'eau, percevoir des taxes d'octroi et procéder à la recherche de nouvelles sources dans les propriétés des particuliers. 
Henri IV mit en projet l'alimentation en eau des quartiers hauts et éloignés, comme Le Louvre et les Tuileries qui purent profiter ainsi de l'eau de Seine grâce à son élévation, due aux premières pompes de la Samaritaine et du Pont Neuf. C'est la Reine, Marie de Médicis, après la mort d'Henri IV, qui fit aboutir ces derniers projets, sous le règne de Louis XIII. Ces nouvelles pompes, à partir de 1608, assuraient un débit de $710 \mathrm{~m}^{3}$ par 24 heures ou 8 litres par seconde.

Afin d'alimenter en eau le palais du Luxembourg qu'elle venait d'acquérir, la Reine fit réaliser le projet de l'aqueduc d'Arcueil avec de nombreuses fontaines et un grand réservoir rue d'Enfer. Cet aqueduc fut construit sur les ruines de l'antique aqueduc de Rungis des Romains et c'est en 1624 que l'eau d'Arcueil arriva en son Palais.

Déjà durant les années 1667-1668-1669 la sécheresse était d'actualité : les aqueducs donnaient peu d'eau et les pompes sur la Seine fonctionnaient tant bien que mal. Aussi Messieurs Jolly et Jacques de Manse, ingénieurs du roi Louis XIV, proposèrent en 1671-1672 et réalisèrent ensuite plusieurs pompes nouvelles à chaque fois améliorées (pompes du Pont Notre-Dame, de Chaillot et du Gros Caillou, chacune surélevant de 30 à 150 litres d'eau par seconde). Ces pompes rendirent de bons services mais elles étaient d'entretien difficile et furent finalement abandonnées et démontées en 1858. Il est raisonnable d'avancer qu'à partir de 1670 , la population parisienne, estimée à 500000 habitants, disposait de près de $3000 \mathrm{~m}^{3}$ par jour d'eaux publiques, soit 6 litres par personne. L'origine de ces eaux était la suivante :

$\begin{array}{ll}\text { Sources du Nord } & : 300 \mathrm{~m}^{3} \text { par jour } \\ \text { Aqueduc d'Arcueil } & : 700 \mathrm{~m}^{3} \text { par jour } \\ \text { Pompe Notre Dame } & : 1500 \mathrm{~m}^{3} \text { par jour } \\ \text { Samaritaine } & : 500 \mathrm{~m}^{3} \text { par jour }\end{array}$

C'est ensuite en 1676, que Pierre-Paul Riquet de Bon Repos et Jacques de Manse présentèrent, au roi Louis XIV et à son conseiller Colbert, un projet qui devait permettre d'amener les eaux de la rivière Ourcq au-dessus du moulin de Mareuil à $18 \mathrm{~km}$ du confluent de la Marne et de la Seine, pour les conduire ensuite jusqu'aux pieds de l'Arc de Triomphe du Faubourg Saint-Antoine. Puis ces eaux emprunteraient un canal jusqu'au fossé de l'Arsenal. Ces eaux auraient comme principal usage, le lavage des rues et des principaux égouts de la capitale, l'embellissement des jardins publics et des maisons Royales. Ce projet fut abandonné à la mort de Colbert et aussi par manque d'argent.

La présence et le fonctionnement, des différentes pompes installées sur la Seine, furent important pour la Ville de Paris puisque c'est 
de cette époque que date la Première Compagnie des Eaux. En août 1776, les frères Perrier proposèrent d'élever l'eau de la Seine et de la distribuer à leurs frais. Louis XVI, en acceptant ce projet le 7 février 1777, autorisait cette nouvelle "Compagnie des eaux" à construire des machines pour élever l'eau de la Seine, à aménager des fontaines dans Paris pour alimenter les petits ménages ou les particuliers et enfin, à placer sous les pavés toutes les conduites nécessaires à la réalisation de ces fontaines. Ces lettres patentes autorisaient donc les frères Perrier à alimenter, à distribuer et à vendre l'eau aux Parisiens. Par abonnement de 3, 6 ou 9 années, le prix était de 50 livres par an pour la fourniture d'un muid (250 l) par jour. Les "petits ménages" pouvaient s'approvisionner aux fontaines publiques pour un prix plus modique.

En 1788 un seul banquier possède toutes les actions de la Première Compagnie des eaux et à la suite de différentes campagnes d'opinions soutenant ou discréditant au contraire cette entreprise, c'est la Ville de Paris, avec l'accord du Roi, qui repris l'ensemble des installations sous la dénomination d'Administration Royale.

Pour puiser l'eau dans la Seine, furent réalisées les pompes à feux de Chaillot, opérationnelles dès 1782 et celles du Gros Caillou en 1786. Des réservoirs furent également construits à Chaillot pour remédier aux à coups de la demande en eau.

Ce fut, en définitive, Napoléon ${ }^{\text {er }}$ qui fit réaliser, entre 1802 et 1825, les travaux du canal de l'Ourcq imaginés antérieurement par Colbert, avec un double objectif de navigation et de ressource pour l'alimentation en eau potable.

Le 15 août 1809 les eaux jaillissent à la fontaine des Innocents. Cette nouvelle installation permettait de livrer quotidiennement, d'une part 2000 pouces d'eau aux maisons et établissements tels que bains, lavoirs ou ateliers industriels et d'autre part 2000 pouces d'eau, également, au service public. L'ensemble des deux services représentait $80000 \mathrm{~m}^{3}$ d'eau chaque jour. Mais la qualité de l'eau distribuée ne va pas de pair avec ces nouvelles et importantes quantités. En effet l'eau est trop chargée en sulfates pour continuer à alimenter tout le secteur privé.

Jusqu'au second Empire les rives de la Seine sont seulement les témoins du développement économique de la ville: navigation marchande, blanchisseries, porteurs d'eau à bretelles.

A l'aube du $19^{\mathrm{e}}$ siècle, il n'y avait dans tout Paris qu'une soixantaine de fontaines dont le débit était interrompu la nuit et il fallait acquitter une somme d'argent pour puiser l'eau. L'ensemble de la distribution d'eau se tourne, alors, vers la desserte à domicile. De nombreux puits privés ou mitoyens furent creusés, 25000 recensés 
en 1833 puis 30000 en 1878 . Mais la plupart des puits, situés dans les vieux quartiers les plus bas sont déclarés, dès 1878, inutilisables du fait de souillures des infiltrations de fosses d'aisance.

La période napoléonienne amène la réalisation de grands projets. En effet Napoléon lui-même voulait une belle capitale, luxueuse avec de belles fontaines féeriques coulant jours et nuits. C'est l'objet du décret de 1806 pour les 65 fontaines existantes et les 15 nouvellement installées.

Au cours des années 1830 se leva un véritable espoir de recueillir des volumes important d'eau par "les eaux jaillissantes" puisées dans la nappe artésienne. La pression exercée dans l'aquifère permet à ces nouvelles eaux de monter jusque dans les quartiers hauts de la capitale. Ces eaux bactériologiquement pures et peu chargées en sels calcaires jaillissent à $28^{\circ} \mathrm{C}$ pour la première fois au puits de Grenelle en février 1841 (900 $\mathrm{m}^{3}$ par jour), ensuite à Passy et par la suite dans bien d'autres puits. 288 sont recensés en 1938. Mais justement à cause de la trop grande exploitation de cette nappe, les puits se tarissent peu à peu. Dès cette époque, le Conseil Municipal de Paris reconnaît que ces eaux sont trop peu abondantes pour qu'elles alimentent tout Paris et de plus qu'elles sont mal adaptées à la consommation domestique du fait de leur saveur.

Quant aux eaux de la Seine pourtant renommées, c'est une première machine à vapeur installée à Austerlitz en 1858 qui permet leur distribution seulement aux quelques fontaines publiques riveraines ou à quelques autres sous concessions privées.

Vers les années 1830 , les ressources en eau de la ville de Paris peuvent être réparties ainsi :

canal de l'Ourcq :

Établissements sur la Seine:

Aqueduc d'Arcueil :

Puits artésien de Grenelle :

Sources du Nord:

Soit au total:
$60000 \mathrm{~m}^{3}$ par jour $19000 \mathrm{~m}^{3}$ par jour $300 \mathrm{~m}^{3}$ par jour $900 \mathrm{~m}^{3}$ par jour $200 \mathrm{~m}^{3}$ par jour $80400 \mathrm{~m}^{3}$ par jour

Un Parisien pouvait ainsi disposer de 61 litres d'eau par jour compte tenu des pertes de l'ensemble du circuit de distribution.

Mais 1832 est l'année de la terrible épidémie de choléra, importé des colonies, qui frappe Paris. Plusieurs facteurs concourent à aggraver ce fléau:

- L'explosion démographique avait rendu inadapté le réseau d'égouts. De même l'entretien des logements et de la voirie n'était 
plus fait dans de bonnes conditions d'hygiène. L'explosion démographique était principalement due à l'exode rural vers les villes industrielles.

- L'hygiène alimentaire était défectueuse. La dénutrition protidique était courante.

- Les problèmes de l'eau potable s'étaient aggravés: cherté pour un besoin autre que vital. Le parisien disposait de seulement 7 litres d'eau par jour (à la même époque, le Londonien en avait 62). L'eau consommée venait du canal de l'Ourcq, de la Seine et des puits. Il n'y avait dans Paris que 217 bornes fontaines au mince filet d'eau. Les eaux de Seine avaient bonne réputation, la population semblait oublier que 60 égouts se déversaient à cette époque dans le fleuve. La pompe à feu de Chaillot était disposée à 50 mètres du Grand Egout parisien dit Turgot, allant de Ménilmontant à Chaillot. Même l'eau provenant des nombreux puits, creusés assez rapidement sans grande précaution, était polluée par les boues noires des pavés, les fuites des fosses d'aisance, et les souillures des cimetières.

Le choléra survient donc dans ces conditions, de mars à septembre 1832 avec deux périodes : l'invasion et la recrudescence. Le choléra se répandit dans 56 départements français et fit 103000 morts environ. Une nouvelle épidémie de choléra se produisit en 1849 faisant à nouveau 110000 morts dans toute la France. Les solutions réelles de meilleures salubrité de la capitale ne furent prises qu'après la troisième épidémie de 1853 qui provoqua encore 9000 morts à Paris.

En 1853 à la veille de la nomination du Baron Hausmann à la Préfecture de la Seine, Paris disposait de $134000 \mathrm{~m}^{3}$ d'eau par 24 heures (100000 $\mathrm{m}^{3}$ provenant du canal de l'Ourcq, 30800 de la Seine, 2400 des sources-dérivations d'Arcueil et du Nord et enfin 800 $\mathrm{m}^{3}$ des puits artésiens. Soit 110 litres par habitant).

Le Préfet Hausmann et l'Ingénieur Belgrand en 1854 proposent pour l'alimentation en eau potable de la Ville de paris la création de deux réseaux, privé pour les particuliers et public pour les bornes fontaines, les fontaines monumentales, les bouches d'incendie et les eaux d'arrosage.

Pour le Baron Hausmann, les eaux de qualité destinées aux particuliers devaient être salubres, limpides et de fraîcheur constante.

Ces deux grandes personnalités, Hausmann et Belgrand, eurent l'inspiration de penser que les eaux chargées en matières organiques dissoutes, originaires de l'Ourcq ou même de la Seine, un peu plus tard, devaient être considérées comme suspectes et insalubres, même si elles gardaient la faveur des Parisiens à l'époque. 
Dans un premier projet, ils attribuèrent ces eaux, celles de l'Ourcq d'abord, puis celles d'Arcueil, des sources du Nord ensuite et enfin celles des puits artésiens à l'usage du service public.

Les eaux de la Seine et des sources du plateau de Champagne furent réservées au service des particuliers.

Le Baron Hausmann et l'Ingénieur Belgrand imposèrent l'individualisation des réseaux de chacun des services, afin d'éliminer les transferts d'eau, d'un réseau à l'autre.

le travail est donc considérable : production d'eau, adaptation des réseaux anciens et création de nouveaux réseaux.

En 1858 les ressources en eau sont les suivantes:

Eaux de l'Ourcq :

Eaux anciennes :

Eaux de sources :

Soit au total :
$105000 \mathrm{~m}^{3}$ par jour $3000 \mathrm{~m}^{3}$ par jour $100000 \mathrm{~m}^{3}$ par jour $208000 \mathrm{~m}^{3}$ par jour

Dans un deuxième projet, le Baron Hausmann fait valoir que les eaux de Seine ne peuvent plus désormais, figurer comme ressources pour l'alimentation en eau de la population et propose de raccorder les conduites du réservoir de Belleville à celles de l'Ourcq. Il est intéressant de citer son propre texte: "les conduites principales partant du réservoir de Belleville rencontreront les conduites maîtresses de l'eau de l'Ourcq et pourront s'emparer aisément de toutes les parties de l'ancien système de distribution qu'on croira devoir attribuer aux nouvelles eaux".

Enfin, peu de temps après, le Baron Hausmann proposera au Conseil Municipal, qui acceptera, de conduire les $100000 \mathrm{~m}^{3}$ d'eau de la Vanne jusqu'à Paris. En 1854 la population de la capitale desservie est de 1700000 habitants. La ville avait doublée de surface et englobait Ménilmontant et Montmartre (partie haute plus difficile à alimenter en eau).

La doctrine d'Hausmann s'inspirait de trois principes importants :

1 - l'affirmation de la maîtrise de la Ville sur la propriété de ses eaux et la gestion de ses services. constante.

2 - Le choix d'eaux de sources pures, salubres et de fraîcheur

3 - La notion d'unité et de complémentarité des deux services, eaux et égouts, rassemblés dans la canalisation complète de Paris "réseaux et galeries souterraines." 
A la suite des propositions précédentes, la dérivation de la Dhuis (cote 128) fut achevée en 1865 et, par l'intermédiaire d'un réservoir à Ménilmontant (cote 108), put alimenter les maisons hautes de Montmartre et de Belleville.

En 1875 ce sont les eaux de sources de la vallée de la Vanne qui arrivent à Paris après un voyage de $173 \mathrm{~km}$ à travers des ouvrages nombreux et onéreux pour la Ville de Paris. Le débit de cette dérivation était de $120000 \mathrm{~m}^{3}$ par jour. Les eaux sont admirables pour leur limpidité, leur fraîcheur constante et un titre hydrotimétrique de 17 à $20^{\circ}$ tout à fait satisfaisant. Ces eaux arrivaient au réservoir de Montsouris d'une capacité de $200000 \mathrm{~m}^{3}$.

En 1878, à la mort de Belgrand, les eaux qui arrivent à Paris se répartissent ainsi :

Eaux de l'Ourcq :

$105000 \mathrm{~m}^{3}$ par jour

Eaux de Seine :

$88000 \mathrm{~m}^{3}$ par jour

Eaux de Marne:

Eaux d'Arcueil et des puits artésiens:

$48000 \mathrm{~m}^{3}$ par jour

Eaux de la Dhuis :

$7000 \mathrm{~m}^{3}$ par jour

Eaux de la Vanne:

$22000 \mathrm{~m}^{3}$ par jour

Total :

$100000 \mathrm{~m}^{3}$ par jour

$370000 \mathrm{~m}^{3}$ par jour

Si les deux services, public et privé, sont considérés comme une seule unité, et si le chiffre de 2 millions d'habitants à Paris est retenu pour cette date, c'est presque 200 litres d'eau qui sont mises à la disposition de chaque individu, soit dix fois plus qu'au début du second Empire.

C'est en 1872 que le célèbre Richard Wallace, l'anglais le plus parisien de son temps offrit à la ville de Paris : "cinquante fontaines à boire, à établir sur les points les plus utiles pour permettre aux passants de se désaltérer" La première fut installée boulevard de la Villette. Elle remporta un si vif succès que la police dut en réglementer l'accès. En quelques années ce fut 208 fontaines qui furent édifiées dans tout Paris. Le grand modèle conçu par le sculpteur CharlesAuguste Lebourg, orné de quatre cariatides supportant un dôme, débite 4000 litres par jour. Le même sculpteur dessina une fontaine applique avec naïade et tritons dont ne subsiste à notre époque qu'un seul exemplaire en service, au coin de la rue Geoffroy-Saint-Hilaire et de la rue Cuvier. Après avoir disparu au fil des ans, les fontaines Wallace, alimentées en eau potable, réapparaissent dans la ville pour couler jour et nuit. 
A partir de 1878, à la mort de Belgrand, c'est Alphand qui prend sa succession et parachève les quelques travaux manquant à la mort de son prédécesseur.

L'essentiel du programme complémentaire après Belgrand va porter sur les eaux de rivières.

Il existe à cette époque en 1878 neuf usines de production d'eau potable à partir des rivières :

- 6 usines à vapeur sur la Seine.

- Chaillot et Austerlitz gérées par la Ville de Paris

- Port à l'Anglais, Maisons-Alfort, Auteuil et SaintOuen gérées par la Compagnie Générale des Eaux. sur la Marne.

- 1 grande usine mixte, hydraulique et à vapeur à St Maur

- 2 usines hydrauliques auxiliaires du canal de l'Ourcq : Trilbardou et Isles.

Vers 1880 l'apport complémentaire en eau, nécessaire à la ville de Paris pour pallier, entre autre, les insuffisances estivales, le manque d'hygiène ou le lavage des rues est estimé à $180000 \mathrm{~m}^{3}$ par jour.

Le programme "eaux de rivières" comprenait les opérations suivantes :

- construction d'une usine d'eau potable à Ivry sur la rive gauche de la Seine. Les eaux sont filtrées puis stérilisées (capacité: $85000 \mathrm{~m}^{3}$ par jour). autre à Bercy

- construction d'une usine d'eau potable à Javel et d'une

- Renforcement de la capacité de traitement de l'usine de St Maur.

La Ville avait alors une capacité de production d'eau potable à partir des rivières de $330000 \mathrm{~m}^{3}$ par jour $\left(240000 \mathrm{~m}^{3}\right.$ avec sept usines sur la Seine et $90000 \mathrm{~m}^{3}$ avec trois usines sur la Marne).

Mais les épidémies antérieures, de choléra et de typhoïde poussent le Conseil d'Hygiène ainsi que la Faculté de Médecine à lancer des appels pour le retour aux eaux de source destinées à la consommation humaine. Un grand emprunt est même lancé plus tard en 1886.

Ainsi, les travaux pour capter les sources de l'Avre, de la Vigne, du Breuil furent mis en chantier de 1891 à 1893. Un aqueduc princi- 
pal long de $102 \mathrm{~km}$ amènent en fin de parcours toutes les eaux au réservoir de St Cloud d'une capacité de $287487 \mathrm{~m}^{3}$. Situé à une cote de $105 \mathrm{~m}$, il pouvait sans difficulté desservir par gravité tous les étages des maisons des quartiers hauts du $14^{\circ}, 15^{\circ}$ et $16^{\circ}$ arrondissements.

A l'occasion de l'exposition Universelle de 1900 et, par conséquent, d'une demande accrue en eau, la dérivation des eaux du Loing et du Lunain furent entreprises. Dans ces deux vallées, de nombreuses sources furent captées dans leurs gîtes géologiques mêmes, ce qui leur procurait encore plus de pureté.

La dérivation de $73 \mathrm{~km}$ était juxtaposée à celle de la Vanne sur une bonne partie du parcours et se terminait dans le réservoir de Montsouris. La capacité de cet aqueduc fut doublée en 1913, il apportait alors $100000 \mathrm{~m}^{3}$ d'eau à Paris.

La dernière grande dérivation, celle de la Voulzie est entreprise en 1917 et terminée en 1925. Les eaux de la Voulzie, comprenant celles du Durteint et du Dragon, sont envoyées par conduite spéciale de $45 \mathrm{~km}$ dans l'aqueduc du Loing dont la capacité de transport avait été prévue à cet effet dès le début de la construction de cet ouvrage. L'appoint en eau de ces 3 sources est de $90000 \mathrm{~m}^{3}$ par jour soit $20 \%$ de l'alimentation de Paris en eau potable.

Tout au long du 20" siècle, le problème de l'eau potable à Paris ne cesse de se poser aux responsables sous deux aspects majeurs:

1 - L'importance des besoins qui vont inexorablement vers l'augmentation.

2 - L'obligation impérieuse de fournir tout au long de l'année une eau de bonne qualité de manière à garantir la santé de ceux qui la boivent, en contre partie d'une pollution de certaines ressources de plus en plus fréquente.

Toujours à l'occasion de l'exposition Universelle de 1900, la Ville de Paris met en œuvre des moyens importants, pour construire les usines de traitement d'eau sur la Seine et la Marne.

L'usine d'Ivry est d'abord réalisée avec une capacité de $80000 \mathrm{~m}^{3}$ par jour (la surface filtrante étant de $14000 \mathrm{~m}^{2}$ ). L'eau est refoulée à l'aide de pompe dans les réservoirs de Ménilmontant et des Lilas.

De 1914 à 1950 la modernisation constante de l'usine a lieu principalement dans trois grands axes.

1 - Amélioration du traitement même de l'eau avec en particulier l'application dès 1914 d'une chloration de l'eau.

2 - L'agrandissement de la surface filtrante. 
3 - Le remplacement des machines à vapeur par des moteurs électriques.

L'usine d'Ivry qui produit $450000 \mathrm{~m}^{3}$ par jour d'eau potable en 1950 verra ce volume constamment diminuer du fait de la dégradation de la qualité de l'eau de Seine et donc du ralentissement de la vitesse de filtration.

A partir de 1976, l'usine est modernisée puis complètement restructurée à partir de 1987 date où la SAGEP, nouveau concessionnaire des installations va instaurer une filière moderne de traitement de l'eau plus axée vers les procédés biologiques.

Sur la Marne, l'usine de Saint-Maur est inaugurée en 1896 et applique comme à Ivry le principe de la filtration lente, mais avec un procédé entièrement nouveau, à base d'ozone, au fort pouvoir oxydant, pour la stérilisation des eaux. Cette usine doit être complètement rénovée dans les années 1995-96-97 avec adjonction de deux procédés, la flottation-coagulation de contact pour éliminer les algues souvent présentes en Marne, et la clarification par décantation pour éliminer la turbidité, deuxième facteur spécifique aux eaux de Marne.

Enfin, la troisième usine de traitement de l'eau de Seine dont la Ville de Paris se dota fut celle d'Orly inaugurée en 1969 avec une autre filière de traitement dite à filtration rapide fondée, entre autre, sur les principes chimiques de coagulation-floculation.

A la fin du 20" siècle, Paris dispose de $800000 \mathrm{~m}^{3}$ d'eau potable par jour soit 200 litres par habitant et par jour. A cela il faut ajouter $40000 \mathrm{~m}^{3}$ d'eau, non potable, destinés au nettoyage des rues et à l'arrosage public.

Les grands réservoirs d'une capacité totale de $1171133 \mathrm{~m}^{3}$ sont au nombre de sept: Ménilmontant (92 $\left.150 \mathrm{~m}^{3}\right)$; Belleville $\left(6358 \mathrm{~m}^{3}\right)$; Montsouris $\left(202591 \mathrm{~m}^{3}\right)$; Les Lilas $\left(208332 \mathrm{~m}^{3}\right)$; Montmartre $(6369$ $\mathrm{m}^{3}$ ); Saint Cloud (425 $973 \mathrm{~m}^{3}$ ); L'Hay-Les-Roses (229 $360 \mathrm{~m}^{3}$ ).

Un des problèmes auquel la Ville de Paris doit faire face est la gestion des ressources afin d'établir un équilibre entre les saisons d'une part (étiage des fleuves en été et crue en hivers-printemps) et d'autre part la consommation plus forte par grosse chaleur, le matin et le soir).

Les projets de construction de barrages sur la Seine, l'Aube, l'Yonne et la Marne ont donc été étudiés dès les premières décennies du 19" siècle. En retenant une partie de l'eau des fleuves et rivières en amont, les barrages ont pour but de réduire l'amplitude des crues (hivers-printemps) et de restituer, ensuite l'eau accumulée, durant la période de sécheresse en été, au fur et à mesure des besoins pour 
permettre aux usines de traitement des eaux de rivières de continuer à fonctionner en toute sécurité.

C'est en 1949 que le premier grand barrage de Pannecière-Chaumard dans la Nièvre a été mis en service $\left(80\right.$ millions de $\left.\mathrm{m}^{3}\right)$. Puis ont été mis en service successivement:

- 1966. Lac de la forêt d'Orient près de Troyes (205 millions de $\mathrm{m}^{3}$ ) C'est une dérivation de la Seine vers une zone inondable (Barrage Seine).

- 1974. Lac du Der Chantecoq sur la Marne près de Saint-Dizier (350 millions de $\mathrm{m}^{3}$ ) (Barrage Marne). Aube).

- 1990. Lac de Brienne sur l'Aube (170 millions de $\mathrm{m}^{3}$ ) (Barrage

L'ensemble de ce dispositif de retenues a parfaitement fonctionné puisque grâce à cela, les années de sécheresse 1989 à 1993 n'ont eu aucune incidence sur le débit de la Seine durant les périodes d'été et par là -même, sur le fonctionnement des usines d'eau potable.

Voici donc l'histoire, de l'alimentation en eau potable de la Ville de Paris, tracée à grands traits et les acteurs d'aujourd'hui, avec les Directions de l'Administration Municipale et les moyens qu'elle met à sa disposition, ne manquent pas de poursuivre le récit moderne de cette passionnante histoire. 